Wilfrid Laurier University

Scholars Commons @ Laurier

Physics and Computer Science Faculty

Publications

Physics and Computer Science

1975

\title{
Multiple Quantum Flux Penetration in Superconducting Loops
}

H.J.T. Smith

University of Waterloo

James A. Blackburn

Wilfrid Laurier University, jabjabjab@cogeco.ca

Follow this and additional works at: https://scholars.wlu.ca/phys_faculty

\section{Recommended Citation}

Smith, H.J.T. and Blackburn, James A., "Multiple Quantum Flux Penetration in Superconducting Loops" (1975). Physics and Computer Science Faculty Publications. 49.

https://scholars.wlu.ca/phys_faculty/49

This Article is brought to you for free and open access by the Physics and Computer Science at Scholars Commons @ Laurier. It has been accepted for inclusion in Physics and Computer Science Faculty Publications by an authorized administrator of Scholars Commons @ Laurier. For more information, please contact scholarscommons@wlu.ca. 


\title{
Multiple quantum flux penetration in superconducting loops*
}

\author{
H. J. T. Smith \\ Physics Department, University of Waterloo, Waterloo, Ontario, Canada \\ J. A. Blackburn \\ Physics Department, Wilfrid Laurier University, Waterloo, Ontario, Canada
}

(Received 3 March 1975)

\begin{abstract}
The dynamics of flux entry into a superconducting loop interrupted by a thin-film Josephson junction have been studied experimentally. A computer simulation of the system yields excellent agreement with these data. It is found that a specific number of flux quanta will bubble into a loop, and that this number depends only on the relative damping which is present.
\end{abstract}

\section{INTRODUCTION}

The behavior of superconducting loops containing single weak links for which $L i_{c} \leqslant \phi_{0}\left(\phi_{0}\right.$ being a flux quantum, $\left.2 \times 10^{-15} \mathrm{~W}\right)$ has been described in some detail by Silver and Zimmerman, ${ }^{1}$ and McCumber. ${ }^{2}$ The observed dependence of circulating current has been used by Jackel, Buhrman, and $W_{e b b^{3}}$ to deduce current-phase relations for superconducting weak links of the point-contact type. Kurkijärvi ${ }^{4}$ considered the thermally driven transitions by such a loop to lower-energy states, although his analysis was given in detail only for $L i_{c} \simeq \phi_{0}$, and high damping. This latter condition essentially restricted the system dynamics so that only one flux quantum could enter at a time. Kurkijärvi did remark that multiple quantum transitions are to be expected for large $L i_{c}$.

Multiple quantum transitions were reported by Sullivan et al. ${ }^{5}$ and elaborated further by Sandell et al. ${ }^{6}$ These experiments involved shunted point contacts coupled to resonant tuned circuits. Generally speaking, the observed transitions involved. 5-10 flux quanta.

Our experimental mechod is intrinsically simpler, requiring no tank curcuits, local oscillators, mixers, and so forth. Circulating loop currents were observed directly, permitting easier interpretation of system dynamics. In addition, very large inductance and capacitance values were achieved by employing macroscopic wire loops and evaporated thin-film Josephson junctions. The associated light damping resulted in transitions involving approximately $10^{5}$ flux quanta.

We have constructed a computer simulation of the system, which agrees quite well with the experiments and predicts the detailed (as yet unobserved) structure in the voltage pulse which is developed across the junction during the multiple quantum transition. The simulation also determines quantitatively the relative number of flux quanta which will penetrate the loop as a function of the system damping.

\section{THEORY}

Suppose a weak link with capacitance $C$, junction tunnel resistance $R$, and maximum dc Josephson current $i_{\sigma}(H)$ interrupts a superconducting loop of inductance $L$. Let $\phi_{x}$ be the external flux which would penetrate the loop if it were open circuited, and let $\phi$ be the actual flux threading the loop. Then $\phi=\phi_{x}-L i$, where $i$ is the net circulating countercurrent

$$
i=i_{c} \sin \varphi+\frac{V}{R}+C \frac{d V}{d t}
$$

$V$ and $\varphi$ are the instantaneous voltage and phase across the weak link.

Using the Josephson relation $d \varphi / d t=2 \mathrm{eV} / \hbar$ and the condition for fluxoid quantization in a thick ring, we obtain

$$
\Phi=\Phi_{x}-\gamma \sin (2 \pi \Phi)-\beta \frac{d \Phi}{d t_{1}}-\frac{d^{2} \Phi}{d t_{1}^{2}},
$$

where $\Phi \equiv \phi / \phi_{0}, \Phi_{x} \equiv \phi_{x} / \phi_{0}, \gamma \equiv L i_{c} / \phi_{0}, \beta=\sqrt{L C} / R C$, and $t_{1} \equiv t / \sqrt{L C}$. We shall be interested in situations for which $\gamma \gg 1$ and at zero temperature. Equation (2) is homologous ${ }^{7}$ to a particle sliding down a sinusoidally modulated parabolic potential surface with velocity-dependent damping. As $\Phi_{x}$ is slowly increased, the particle is carried up the parabola in a potential well which becomes progressively more shallow. Finally, at some critical $\Phi_{x c}$, the potential barrier goes to zero, and the particle is released to roll down one or more "bumps" until it is finally captured in a state with a new $\Phi$. For sufficiently large damping, it will be arrested at the next-lowest state, whereas for small damping, a multiple-state transition may occur. In order to investigate these properties quantitatively, we have constructed a computer simulation $^{8}$ of Eq. (2). Solutions are not sensitive to the choice of initial conditions - we used $(\Phi)_{0}=0$, $\left(d \Phi / d t_{1}\right)_{0}=0$, and $\Phi_{x}=\gamma\left(\simeq \Phi_{x c} ;\right.$ that is, $\phi_{x c} \simeq L i_{c}$ when $\left.L i_{c} \gg \phi_{0}\right)$. Occasionally, $(\Phi)_{0}=-1$ was necessary to force at least one transition, because at 


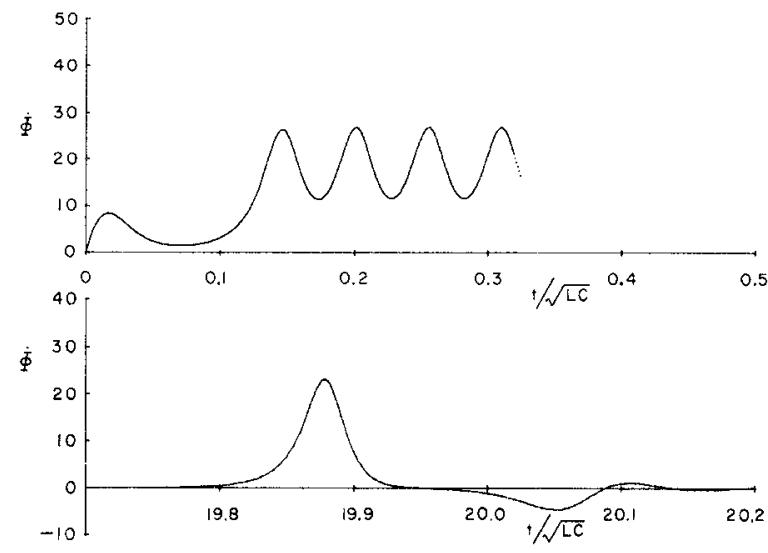

FTG. 1. Time derivative of loop flux for $\gamma=1.000 ; \beta$ $=50$. In effect, this represents the junction voltage as a function of time. Note the break in the time scale. The entry of the first three and last flux quanta into the loop is clearly seen, as is the fact that quantum-transit time is somewhat longer for the final entries. Note that the mean-flux velocity is approximately $\gamma / \beta=20$.

$\Phi_{x}=\gamma$, a very slight potential well remained. Setting $(\Phi)_{0}=-1$ is equivalent to rolling the particle a little further up the parabola before release.

In a typical simulation, it was found that a specific number of flux quanta ( $\Phi_{\text {enter }}$ ( would bubble through the weak link into the loop. Figure 1 illustrates this behavior for parameter values $\gamma=1000, \beta=50$.

Since $V=\left(\phi_{0} / \sqrt{L C}\right) \dot{\Phi}$, this plot directly gives the

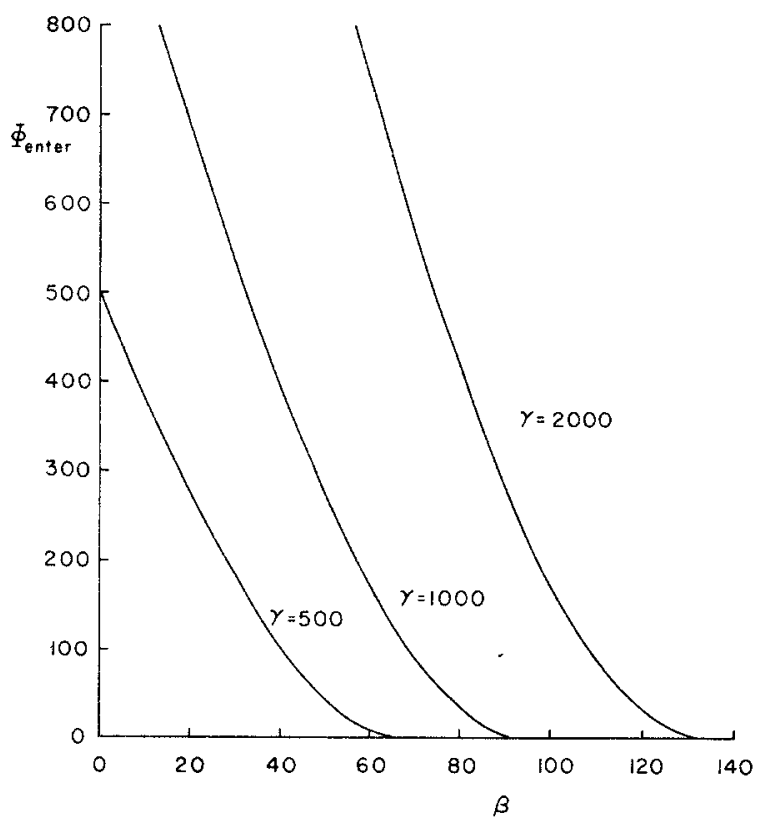

FIG. 2. Plot of the number of flux quanta which enter a superconducting loop containing a Josephson junction as a function of the damping of the circuit.

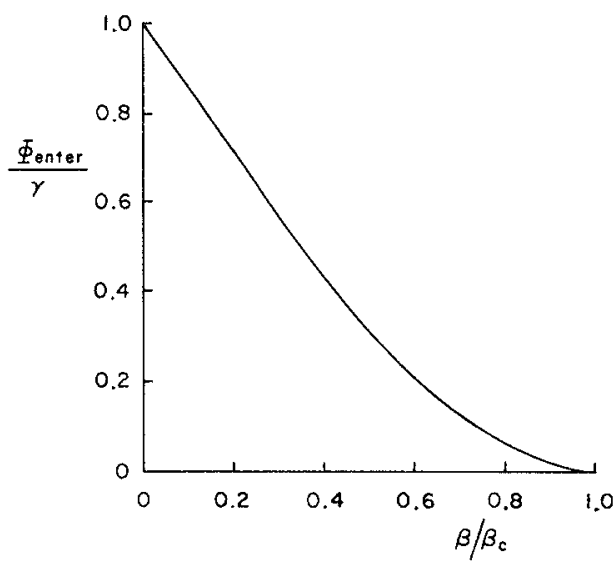

FIG. 3. Universal curve, which may be extracted from Fig. 2.

time-dependent voltage across the Josephson device. As each quantum of flux enters the loop, the order-parameter phase slips by $2 \pi$.

Figure 2 illustrates the dependence of $\Phi_{\text {enter }}$ on $\beta$ and $\gamma$. Note that as $\beta \rightarrow 0, \Phi_{\text {enter }} \rightarrow \gamma$ and that for $\beta \geqslant \beta_{c}, \Phi_{\text {enter }} \leqslant 1$ (the system is overdamped and so cannot overshoot the next-lower-available state). A "universal" curve may be obtained by replotting $\Phi_{\text {enter }} / \gamma$ versus $\beta / \beta_{c}$, and this is shown in Fig. 3 .

\section{EXPERIMENTAL RESULTS AND COMPARISON WITH THEORY}

A thin-film Sn-SnO-Sn Josephson junction $\left(0.013 \times 0.013 \mathrm{~cm}^{2}\right)$ was evaporated onto a glass substrate. This junction was made part of a lead ring, as shown in Fig. 4, with lead pressure contacts

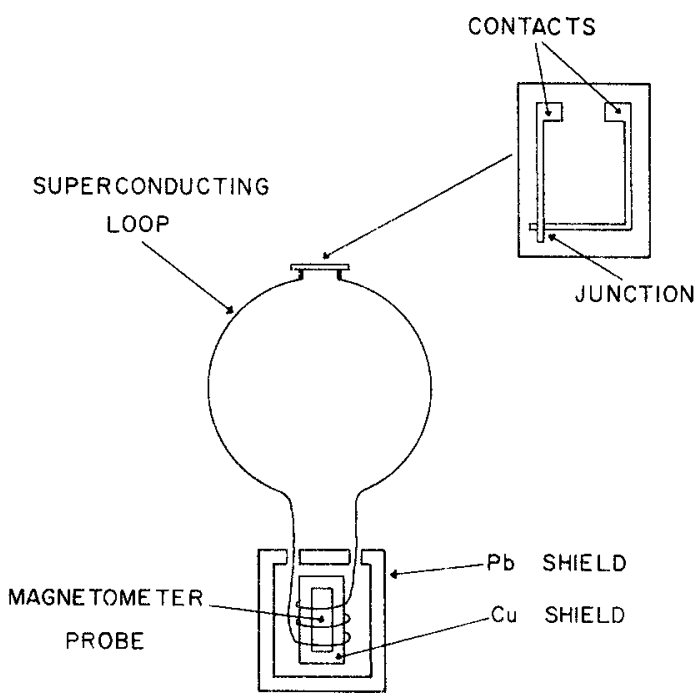

FIG. 4. Schematic diagram of the experimental configuration showing the superconducting loop, magnetometer and shields, and thin-film junction-all of which are immersed in liquid helium. 


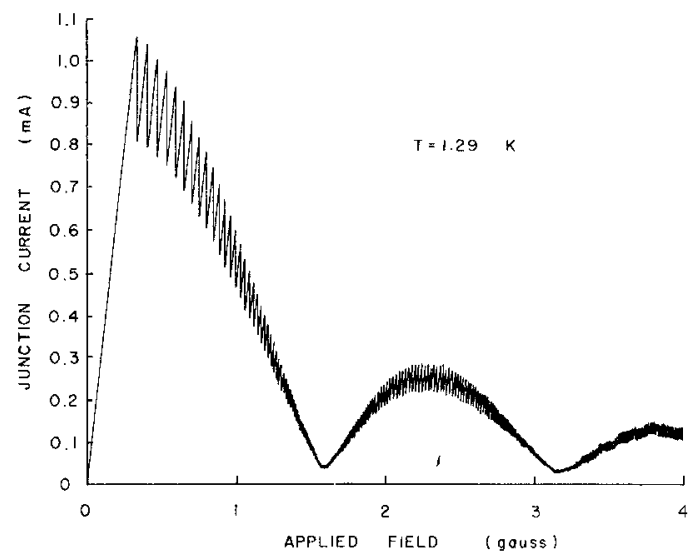

FTG. 5. Typical experimental record showing $25 \%$ vertical transitions each time the junction-critical current is exceeded, at which point a series of flux quanta bubble into the loop. Note the uniformity of these transitions and the quality of the Fraunhofer envelope.

between the ring and the glass slide. The circulating loop current was detected by means of a zeroresistance ammeter, also shown in Fig. 4. A doubly-shielded fluxgate magnetometer probe (Hewlett-Packard No. 428B, probe No. 3529A) was placed inside a nine-turn coil formed from a portion of the lead-loop wire. The copper shield prevented the $40-\mathrm{kHz}$ probe bias signal from coupling into the superconducting loop, while the lead shield excluded external magnetic fields.

The external field was arranged to be perpendicular to the plane of the superconducting loop. The field coil current was linearly increased with time.

An experimental result is presented in Fig. 5 . As expected for large $\gamma$, the circulating loop cur rent increases linearly with external field until the critical-junction current is reached. At this point, an abrupt transition occurs as the system admits $\simeq 0.25 \gamma$ flux quanta. The total transition time $\tau$ may be estimated from the fact that a terminal-flux velocity is given approximately by $\gamma / \beta$. One flux quantum thus takes about $(\beta / \gamma) \sqrt{L C}$ seconds to enter the loop; hence, $\tau \simeq 0.25(L / R)$, which is of the order of $10^{-6}$ sec. The experi- mental record is quite consistent with such a rapid transition.

As the external field continues to increase, the circulating loop current rises again with a slope equal to the original rate of increase. A succession of $25 \%$ transitions and current ramps follows. The Fraunhofer modulation envelope is a familiar feature of non-self-field-limited Josephson junctions.

For the loop and thin-film junction employed, we estimate the following values: $L=10^{-6} \mathrm{H}, R=0.7 \Omega$, $i_{c}=1.09 \mathrm{~mA}\left(\gamma=5 \times 10^{5}\right)$. From the simulations, we find $\beta_{c}=2.97 \sqrt{\gamma}$, and so $R \sqrt{ } C=0.337\left(\beta / \beta_{c}\right)^{-1}$ $\left(\phi_{0} / i_{c}\right)^{1 / 2}$. For this junction, $\Phi_{\text {enter }} / \gamma=0.25$, and thus from Fig. 3, $\beta / \beta_{c}=0.55$. At $H=0, i_{c}=1.09$ $\mathrm{mA}$, and so $C \sim 1.5 \times 10^{-12} F$, a reasonable value for this type and size of junction.

As $H$ is increased, $i_{c}(H)$ varies with the usual Fraunhofer modulation. As a result, $\gamma$ will vary. The effect of the magnetic field is analogous to eliminating some portion of the total junction area from carrying current. Since $i_{c}(H) \propto A$, (where $A$ is the effective junction area) $C \propto A$ and $R \propto 1 / A$, we conclude that the relative damping $\beta / \beta_{c}$, which is itself proportional to $\left(R \sqrt{i_{c} C}\right)^{-1}$, will be unchanged. This is confirmed by experiment. In Fig. 5, the fractional decrease in circulating current at each transition remains at $25 \%$ for all values of $H$.

\section{CONCLUSIONS}

We conclude that $\mathrm{Eq}$. (2) represents a very good theoretical description of superconducting loops broken by Josephson junctions. Thin-film devices with their associated large capacitance (compared to point contacts) produce a light-damping condition in which multiple-quantum-flux entry occurs. Computer simulations produce accurate predictions as to the number of flux quanta which will bubble into the loop.

\section{ACKNOWLEDGMENTS}

The authors wish to acknowledge extensive experimental assistance from $V$. Keith and also are grateful to the Computer Centre, Wilf rid Laurier University for a grant of computer time.
* Supported by National Research Council of Canada.

${ }^{1}$ A. H. Silver and J. E. Zimmerman, Phys. Rev. 1.57, 317 (1967).

${ }^{2}$ D. E. McCumber, J. Appl. Phys. 39, 2503 (1968).

${ }^{3}$ L. D. Jackel, R. A. Buhrman, and $W$. W. Webb, Phys. Rev. B 10, 2782 (1.974).

${ }^{4}$ J. Kurkijarvi, Phys. Rev. B $\underline{6}, 832$ (1972).

${ }^{5}$ D. B. Sullivan, R. L. Peterson, V. E. Kose, and J. E. Zimmerman, J. Appl. Phys, 41, 4865 (1.970).
${ }^{6}$ R. D. Sandell, J. P. Wikswo, Jr., J. M. Pickler, and B. S. Deaver, Jr., J. Appl. Phys. 44, 3312 (1.973).

${ }^{7}$ L. D. Jackel, W. W. Webb, J. E. Lukens, and S. S. Pei, Phys. Rev. B 9, 115 (1.974).

${ }^{8}$ A standard fourth-order Runge-Kutta technique was used. See, for example, Donald Greenspan, Discrete Numerical Methods in Physics and Engineering (Academic, New York, 1974), p. 40. 